\title{
THREE-POINT METHOD FOR FAST AND ROBUST FIELD MAPPING FOR EPI GEOMETRIC DISTORTION CORRECTION
}

\author{
Pelin Aksit, John A. Derbyshire, Jerry L. Prince \\ Global Applied Science Laboratory, GE Healthcare; Laboratory of Cardiac Energetics, NHLBI, \\ National Institutes of Health; Image Analysis and Communications Laboratory, Electrical and Computer \\ Engineering, Johns Hopkins University
}

\begin{abstract}
Because of its superb temporal resolution, echo planar imaging (EPI) is widely used for functional magnetic resonance imaging (fMRI), diffusion tensor imaging (DTI), and monitoring dynamic processes. EPI suffers from geometric distortions caused by magnetic field inhomogeneities, and although these distortions can be corrected by field mapping, current methods require lengthy auxiliary image acquisitions and/or phase unwrapping. We present a 3-point method for field mapping that circumvents these problems and is robust to additive noise. Results demonstrate that the performance of this approach is markedly better than the standard twopoint method and is comparable to methods requiring much longer auxiliary image acquisitions.
\end{abstract}

Index Terms - Magnetic resonance imaging, error correction, phase distortion, phase measurement

\section{INTRODUCTION}

Conventional magnetic resonance imaging acquires a small number of lines of k-space data after each RF excitation, and the excitations are repeated many times in order to cover all of k-space. In EPI, the complete k-space is acquired following a single RF excitation using a train of gradient recalled echoes. Despite the use of larger rapidly switched gradients and typically lower resolution in EPI, the acquisition of all of k-space requires a longer readout period compared to conventional sequences. The bandwidth per pixel in the phase encoding direction is low, making EPI more susceptible to artifacts in this direction. Off-resonance effects, from local inhomogeneities at intersections of samples of different susceptibilities, and main field inhomogeneities, cause additional phase accumulation over the long readout period.

Since these effects are spatially varying, geometric distortions arise and image voxels are compressed or

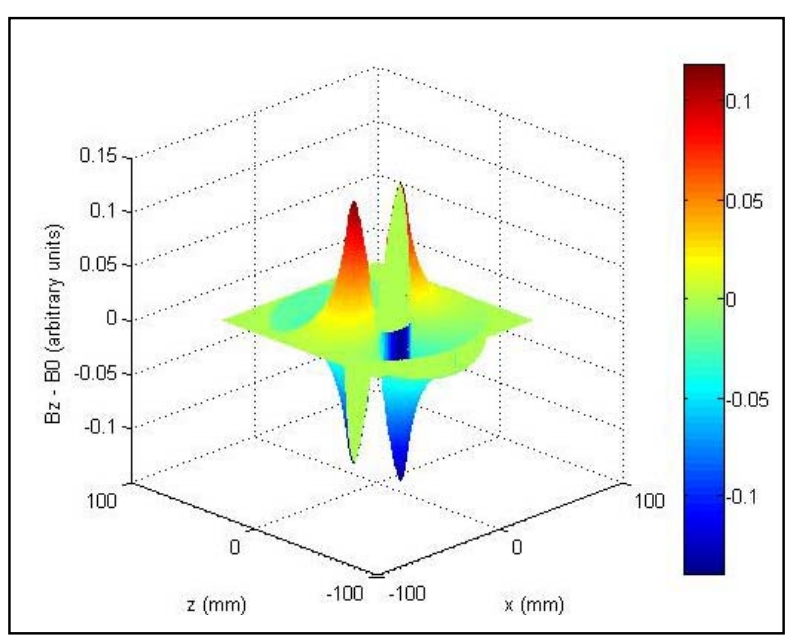

Figure 1: Field pattern within the water-filled space of the system, analytical solution.

stretched depending on the local field gradients that they experience. Information from distorted voxels appears in the wrong place, and signal intensity is darker or brighter because of volume distortion. Geometric distortion scales linearly with main field strength and is most prominent near paranasal sinuses, anterior orbits, the skull base, the liver-lung interface, and air containing bowel loops. Geometric distortion hinders accurate registration of EPI data sets with functional information (i.e. BOLD) or structural information (white matter tracts) to anatomically correct non-EPI data sets.

Numerous techniques have been proposed for geometric distortion correction. These include field mapping [1][2], multi-reference methods (multi-echo, PSF mapping) [3][4], reversed gradient [5], real-time [6] methods and post-processing [7]; each has different tradeoffs between additional acquisition time for distortion correction and accuracy of the correction. Multireference techniques typically measure distortion accurately however they require long acquisition times ( $N_{y} / 4$ to $2 N_{y}$ additional data sets where $N_{y}$ is the number of phase encode lines in the EPI). Reversed 

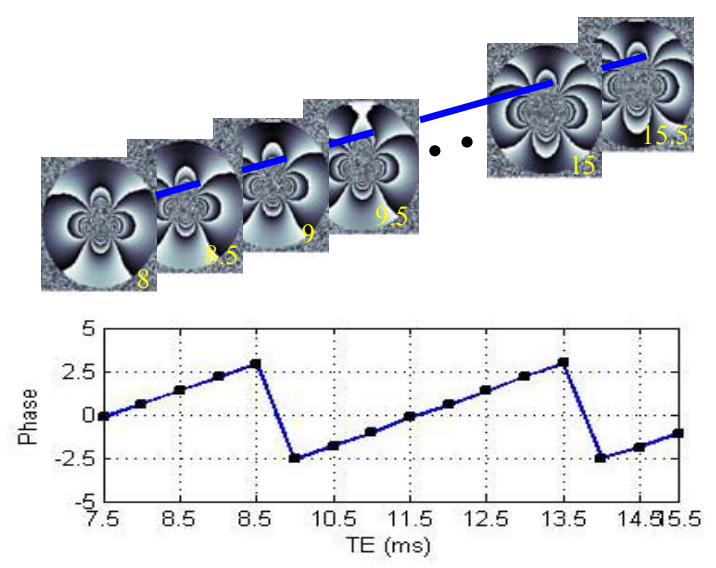

Figure 2: Phase images and sample phase evolution with increasing echo time in an image pixel

gradient techniques which acquire each data set twice in opposite directions to deduce the translation and intensity correction are more susceptible to noise in the data and suffer from streaking artifacts. Real-time or single-shot measure phase differences between multiple acquisitions following a single RF pulse, however, their resolution is limited by signal decay, especially at high fields.

The most commonly used correction approach has been field mapping, which measures variations in the magnetic field to calculate local pixel shifts in the image. The magnetic field map at each pixel is calculated from the slope of phase accumulation over time, as depicted in Figure 2. There are two main approaches to field mapping. The first approach [1] acquires two images at different echo times, and calculates the field map from the phase difference between these. If these two echo times are far apart, the amount of phase accumulation exceeds $2 \pi$ and phase unwrapping is needed. Phase unwrapping is problematic at regions of high susceptibility and disconnected regions. If the two echo times are selected close to each other in order to avoid phase unwrapping, the slope measurements are strongly influenced by the noise in the phase measurements, and typically multiple excitations [1] are used to get an accurate fieldmap. The second approach [2] acquires several images at different echo times, fits a line to the phase accumulation over time and calculates the field map from the slope of the fit, increasing the time needed for field mapping. Obtaining a robust field map continues to be challenging.

In our approach, the field map is obtained from a 3point acquisition. The first two acquisitions have closely spaced echo times and the third acquisition has a significantly longer echo time. This combines the accuracy of the linear fit method (using significantly fewer acquisitions) with the reduced acquisition time of the 2-point method (without the need for phase unwrapping).

\section{METHOD}

To investigate different approaches to field mapping, we built a phantom comprising concentric tubes filled with air and water for which the field distribution is known analytically. The inner tube of inner radius $r_{1}$ and outer radius $r_{2}$ was filled with air; the space between the tubes was filled with water.

The phantom was scanned on a GE 3T Excite MRI scanner with $150 \mathrm{mT} / \mathrm{m} / \mathrm{ms}$ and $40 \mathrm{mT} / \mathrm{m}$ slew rate and gradients (GE Healthcare, Waukesha WI), and a quadrature head coil using an RF spoiled gradient recalled echo pulse sequence, with the following parameters: 256x256, 20cm FOV, $10 \mathrm{~mm}$ slice thickness, $\mathrm{TR}=50 \mathrm{~ms}$, flip $=30$, and $\mathrm{BW}=16 \mathrm{kHz}$, requiring $12.8 \mathrm{~s}$ per slice. A series of 16 images were acquired with the range of echo times $\mathrm{TE}=8 \mathrm{~ms}$ to $\mathrm{TE}=15.5 \mathrm{~ms}$ with $0.5 \mathrm{~ms}$ steps.

Five methods for generating field maps using 2, 3 or all 16 of the images were implemented. In method 1 all 16 data points were used. The phase evolution over the closely spaced data points was unwrapped and the slope of phase versus echo time was calculated from a linear least squares error fit. The field map was obtained by scaling this slope according to $\Delta B=$ slope $/(2 \pi \cdot \gamma)$.

Method 2 also used all 16 data points. In this case, the phase evolution over time was determined by a Fourier transform along the temporal dimension. The linear phase caused a shift in the peak of the spectrum. The field map was obtained by scaling the amount of pixel shift in the spectrum: $\Delta B=\left(n_{-}\right.$pixels_shift $\left./ N\right) /(\gamma . \Delta T E)$ where $N$ is the Fourier transform size, in this case 128.

Three-point field mapping was implemented using two approaches. In method 3 , the data points are used as two pairs, one to provide dynamic range and the other for robustness to noise. The amount of wrap in the last data point was identified using the phase accumulation between the first two closely spaced data points: $k=\operatorname{round}\left(\arg \left(\phi_{2} \phi_{1} *\right) \cdot(\right.$ nsteps $\left.)-\arg \left(\phi_{16} \phi_{1} *\right)\right) /(2 \pi)$ and the field map generated using the unwrapped phase: $\Delta B=\arg \left(\phi_{16 \text { unwrapped }} \phi_{1} *\right) /(2 \pi . \gamma . n s t e p s . \Delta T E)$, where nsteps $=15$.

Method 4, the second 3-point field mapping method, is similar to method 2. It determines the amount of phase accumulation over echo times from the spectrum shift it causes in the temporal frequency domain, via a Fourier transform, and calculates a field map as in method 2 . 
Method 5 uses only the first two data points, and a field map is generated according to $\Delta B=\arg \left(\phi_{2} \phi_{1} *\right) /(2 \pi \cdot \gamma \cdot \Delta T E)$.

Gaussian noise was added to the experimental data and method 1 was used as a gold standard to evaluate the accuracy of other methods under different signal and noise levels.

\section{RESULTS}

The field distribution in the water region of the model is: $\mathrm{B}_{\mathrm{z}}=\left(1+\chi_{w}\right)\left[1-\frac{\chi_{w}}{2}+\frac{\left(\chi_{\text {eff }}-\chi_{w}\right)}{2} \frac{r_{1}^{2}}{r^{2}}\left(\frac{2 z^{2}}{r^{2}}-1\right)\right] B_{0}$ where $\chi_{w}$ and $\chi_{e f f}$ are the susceptibility of water and effective susceptibility of the inner tube, respectively [8].

The analytical solution is shown in Figure 1 is in good agreement with measured field distributions. The maximum field variation was measured as 0.1108 Gauss, corresponding to approximately $3.7 \mathrm{ppm}$ at $3 \mathrm{~T}$, or 472 $\mathrm{Hz}$.

Field distributions calculated using methods 2 to 5 were compared to that of method 1. For qualitative evaluation, the results are shown in Figure 3 for high and low SNR cases. Method 2 has most of its errors along the phase discontinuities, it is robust to noise, and works well at edges. Method 3 smoothly follows the field when SNR is high, except at the susceptibility interface where it has its largest errors. Method 4 behaves similar to methods 2 and 3 , it has most of its errors at the large susceptibility interface and along phase discontinuities. Method 5 also has many errors along the large susceptibility interface, and is the noisiest method evaluated.

Table 1 summarizes the error performance of Methods 2 through 5 using Method 1 as the truth, averaged over 10 repetitions. As expected, both of the 3-point methods always perform better than the 2-point method and worse than the 16-point methods. The 3-point methods provide considerable improvement in accuracy over the 2-point method with a modest increase in scan time, for a fraction of the acquisition time compared to the 16-point method, especially when SNR is 10 or better. Among the 3-point methods: the Fourier approach of method 4 performs slightly better when SNR is high $(>30)$; and the 2-level phase unwrapping approach performs better at lower SNRs.

\section{DISCUSSION AND CONCLUSION}

We presented fast and robust field 3-point field mapping method. With this approach, the effects of noise are supressed by using the $3^{\text {rd }}$ data point which may have considerable phase wrap without an adverse effect. The closely spaced points maintain dynamic range. There should be no wrap between the closely spaced points.

The phantom had considerably larger susceptibility distortion $(+/-500 \mathrm{~Hz})$ than that is expected in the human body $(+/-200 \mathrm{~Hz})$, so further studies should be carried out to study the impact of this approach in-vivo.

\begin{tabular}{|c|c|c|c|c|}
\cline { 2 - 5 } \multicolumn{1}{c|}{} & \multicolumn{4}{c|}{ Average error per pixel } \\
\hline SNR & $\begin{array}{c}\text { 16 PT } \\
\text { Method } \\
\mathbf{2}\end{array}$ & $\begin{array}{c}\text { 3PT } \\
\text { Method } \\
\mathbf{3}\end{array}$ & $\begin{array}{c}\text { 3PT } \\
\text { Method } \\
\mathbf{4}\end{array}$ & $\begin{array}{c}\text { 2PT } \\
\text { Method } \\
\mathbf{5}\end{array}$ \\
\hline 34.34 & 0.00097 & 0.00138 & 0.00110 & 0.00205 \\
\hline 33.73 & 0.00098 & 0.00142 & 0.00133 & 0.00328 \\
\hline 19.44 & 0.0098 & 0.00150 & 0.00178 & 0.00414 \\
\hline 15.06 & 0.0099 & 0.00167 & 0.00233 & 0.00484 \\
\hline 12.72 & 0.0099 & 0.00196 & 0.00292 & 0.00547 \\
\hline 11.22 & 0.00100 & 0.00235 & 0.00348 & 0.00602 \\
\hline 10.14 & 0.00100 & 0.00278 & 0.00405 & 0.00652 \\
\hline 9.32 & 0.00101 & 0.00327 & 0.00459 & 0.00699 \\
\hline 8.67 & 0.00101 & 0.00376 & 0.00510 & 0.00743 \\
\hline 8.14 & 0.00101 & 0.00425 & 0.00562 & 0.00784 \\
\hline
\end{tabular}

Table 1: Effect of SNR on field mapping error

\section{REFERENCES}

[1] P. Jezzard, and R.S. Balaban, "Correction for Geometric Distortion in Echo Planar Images from B0 Field Variations," MRM, 34: pp.65-73, 1995.

[2] P.J. Reber, E.C. Wong, R.B. Buxton, and L.R. Frank. "Correction of Off Resonance-Related Distortion in EPI Using EPI-Based Field Maps,” MRM, 39: pp.328-330, 1998.

[3] N. Chen, and A.M. Wyrwicz. "Correction for EPI Distortions Using Multi-Echo Gradient-Echo Imaging," MRM, 41: pp.1206-1213, 1999.

[4] H. Zeng and R. T. Constable. "Image Distortion Correction in EPI: Comparison of Field Mapping With Point Spread Function Mapping," MRM, 48: pp. 137-146, 2002.

[5] N. Voss, and A.M. Wyrwicz. "Correction for EPI Distortions Using Multi-Echo Gradient-Echo Imaging," $M R M$, 41: pp.12061213,1999

[6] V. Roopchansingh, R.W. Cox, A. Jesmanowicz, B.D. Ward, and J.S. Hyde. "Single-shot magnetic field mapping embedded in echo-planar time-course imaging," $M R M, 50(4)$ : pp.839-843, 2003.

[7] N. Chen, K. Oshio, and L. P. Panych. "Application of kspace energy spectrum analysis to susceptibility field mapping and distortion correction in gradient-echo EPI," $N I$, 31(2): pp.609-622, 2006.

[8] A. J. Lucas, S. J. Gibbs, E. W. G. Jones, M. Peyron, J. A. Derbyshire, L. D. Hall. "Diffusion Imaging in the presence of Static Magnetic-Field Gradients," JMR, 104: pp.273-282, 1993. 


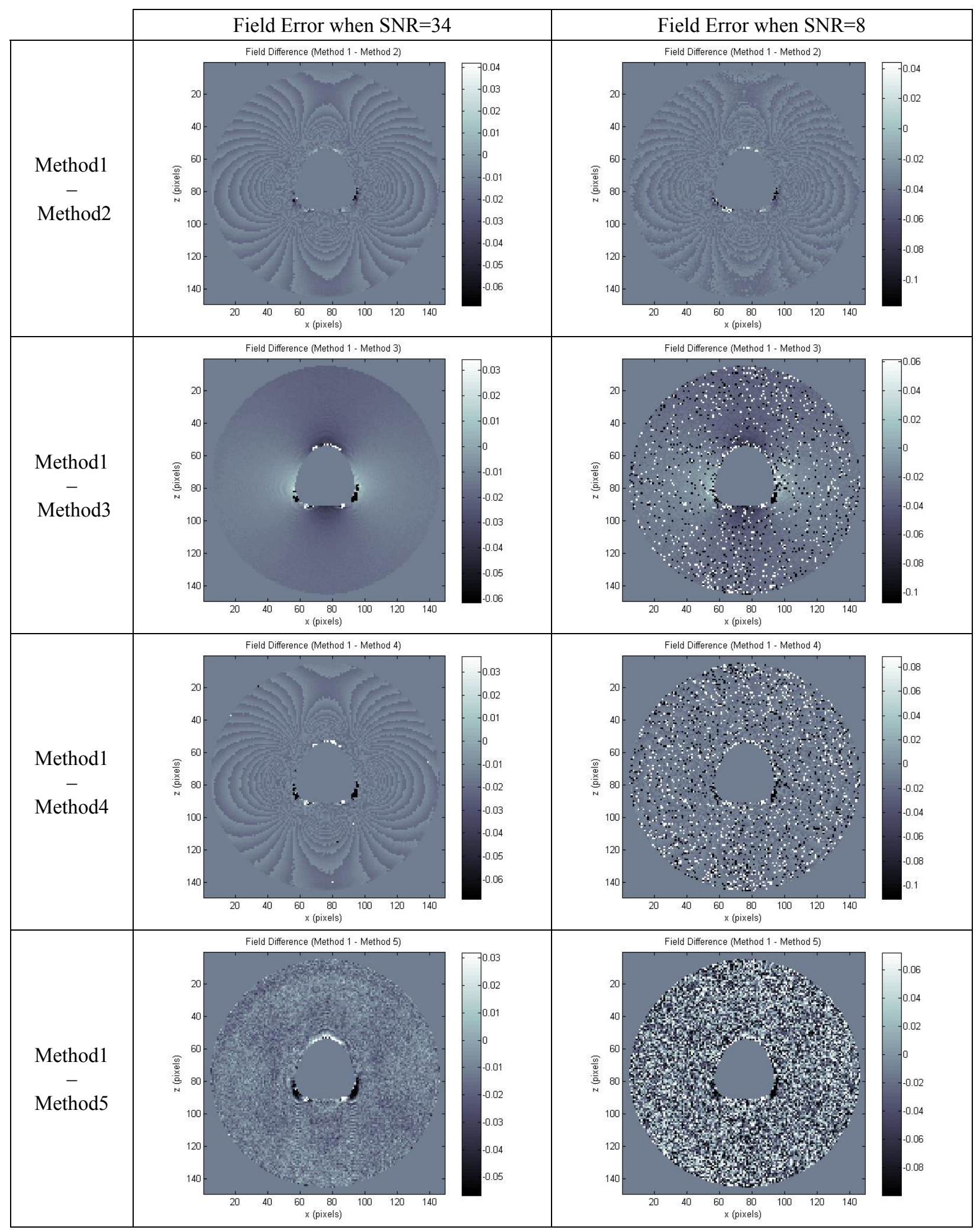

Figure 3: Comparison of residual errors in field mapping methods under high and low SNR conditions. 